\title{
The Importance of Prostate-Specific Membrane Antigen Expression in Carotid Body Paragangliomas
}

\author{
Original Investigation \\ ๑ Hasan Yasan ${ }^{1}$, ๑ Yusuf Çağdaş Kumbul1 , ๑ İbrahim Metin Çiriş², \\ ๑ Mehmet Emre Sivrice ${ }^{1}, \oplus$ Erdoğan Okur $^{1}$ \\ ${ }^{1}$ Department of Otorhinolaryngology \& Head and Neck Surgery, Süleyman Demirel University Faculty of Medicine, \\ Isparta, Turkey \\ ${ }^{2}$ Department of Pathology, Süleyman Demirel University Faculty of Medicine, Isparta, Turkey
}

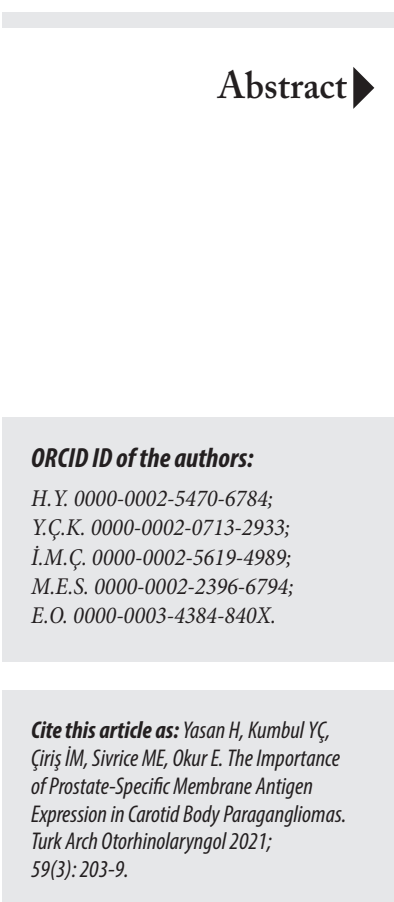

Corresponding Author:

Hasan Yasan; dryasan@hotmail.com

Received Date: 13.04.2021 Accepted Date: 04.06 .2021

Content of this journal is licensed under a Creative Commons Attribution 4.0 International License. Available online at www.turkarchotolaryngol.net

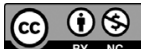

DOI: 10.4274/tao.2021.2021-3-17

Objective: Prostate-specific membrane antigen (PSMA) is a transmembrane protein expressed in prostate cancer. It is, however, also expressed in the neovasculature of some non-prostatic solid tumors. Carotid body paragangliomas (CBPs) are highly vascular neoplasms. In this study, we aimed to investigate the possible role of PSMA expression in CBPs. There are no studies in the literature that report to have investigated the relationship between PSMA and CBPs.

Methods: This study is a retrospective analysis of cases diagnosed with CBP based on their demographic, clinical, radiological, surgical and immunohistochemical findings. Immunohistochemical examination results of Ki-67, S100, synaptophysin, chromogranin were retrieved from patient files. Then, the paraffin blocks of CBPs specimens, stained by PSMAantibody by immunohistochemical methods were examined histopathologically.

Results: The number of patients operated on for CBP was 12 (four men and eight women). Ten out of 12 specimens were suitable for staining and histopathological examination. Capsular and/ or vascular invasions of tumors were seen in complicated cases. Intratumoral vascular PSMA expression was seen in all specimens except one. Extratumoral vascular PSMA expression was not detected in any of the cases. Tumoral cell PSMA staining was seen in six of ten cases.

Conclusion: We found higher intratumoral vascular expressions of PSMA nearly in all CBPs, but we could not assess the statistical significance because of the small number of specimens. These data might be a guide for future studies that are planned for either diagnostic or therapeutic approaches to CBPs.

Keywords: Carotid body, carotid body tumor, immunohistochemistry, paraganglioma, prostatespecific membrane antigen, surgery, histopathology

\section{Introduction}

While carotid body paragangliomas (CBPs) are uncommon tumors, they account for the majority of the paragangliomas (PGLs) in the head and neck (60-70\%) (1). Patients with persistent hypoxia and complaints like chronic obstructive pulmonary disease, congenital cyanotic heart disease, and 
those living in high-altitude areas are at risk (especially women) $(2,3)$. Immunohistochemistry shows that PGLs are positive for neuroendocrine markers such as chromogranin and synaptophysin (3).

Prostate-specific membrane antigen (PSMA) is a transmembrane protein that is found in the neovasculature of some non-prostatic solid tumors, as well as of prostate cancers. PSMA expression is not only found in prostate epithelial cells, but also in tumor-associated endothelial cells in a variety of solid tumors (4). If surgery is difficult or impossible to perform, in vivo PSMA expression in tumors can serve as a deputy biomarker for angiogenesis, and this rationale can be used for non-invasive targeted therapy in solid vascular tumors such as head and neck PGLs (5).

The target is labeled with a diagnostic radionuclide, and once expression is confirmed, the same target is labeled with a therapeutic radionuclide, and the procedure is carried out. Choudhury and Gupta (6) reported the treatment of neuroendocrine tumors with 177-lutetium (Lu-177) by this mechanism. Expressions of new molecules may give rise to new imaging and/or treatment modalities. To that end, PSMA may be a promising novel target in CBPs. We investigated the staining pattern and other findings of CBP cases that were operated on in our clinic. This study is the first to investigate the relationship between PSMA and CBPs.

\section{Methods}

The files of 12 patients (four men and eight women) who were diagnosed with CBP and operated on in Süleyman Demirel University Faculty of Medicine, Ear Nose and Throat Clinic from January 2000 to January 2021 were analyzed. The Clinical Research Ethics Committee of Süleyman Demirel University approved our study protocol. (approval date: 11/09/2020; approval number: 254). The study was designed as a retrospective study; therefore, informed consent was not obtained from the patients.

Files of all patients that were diagnosed with CBP were thoroughly examined. The findings retrieved from patient files were: clinical examination results (horizontally mobile but vertically fixed mass in the carotid triangle, brown skin patches, etc.), computed tomography (CT) images, magnetic resonance images (MRIs) of the neck, and angiography of the carotid system, operational findings, and histopathological records. CT,MRI, and angiographic images were re-evaluated from the recordings. Immunohistochemical investigation results of Ki-67, S100, synaptophysin, chromogranin were retrieved from the reports in patient files. All findings were compared and interpreted.

All patients were routinely followed up four times (every three months) in the first year and annually in the following years. The basic follow-up consisted of routine physical examination and MRI. After evaluating all files, paraffinembedded blocks were taken from the archives of the pathology department. Immunohistochemical staining and examination were done using PSMA antibody.

\section{PSMA Immunohistochemistry}

PSMA antibody was applied to the specimen blocks via immunohistochemical method in the hematoxylin and eosin (H\&E) stained sections. PSMA antibody (clone 3E6, isotype: $\mathrm{IgG1}$, kappa, DAKO flex ready to use monoclonal mouse antibody provided in liquid form in a buffer containing stabilizing protein and $0.015 \mathrm{~mol} / \mathrm{L}$ sodium azide; Dako, California, USA) was prepared according to the manufacturer's instructions as expressed in the data sheet. The tissue samples that were obtained from the formalinfixed paraffin-embedded blocks and taken to four $-\mu \mathrm{m}$ thickness adhesive-coated slides were processed together with prostate tissue sample as antibody positive control and the sample sent alongside as negative control to the automated immunohistochemistry device.

\section{Evaluation of Immunohistochemical Staining}

Immunohistochemically stained slides were evaluated by the same experienced pathologist. PSMA immunohistochemically stained slides were examined under light microscope and accepted as positive cytoplasmic staining and/or positive cytoplasmic membrane staining.

The vascular structures in the epithelial and stromal foci of the tumor were identified from H\&E-stained tumor sections and taken to the simultaneously stained PSMA. Vascular percentage score (expression score) was defined as the number of cells stained by PSMA throughout counted cells. The intensity score was defined as the staining intensity of the cells examined.

Vascular percentage score was defined as Score $0=0 \%-5 \%$; Score $1=5 \%-50 \%$, and Score $2=>50 \%$. The intensity score was defined as Score $0=$ none-weak, Score $1=$ moderate, and Score $2=$ strong. PSMA expression was scored according to the intensity of the tumor epithelium, as Score $0=$ noneweak, Score 1 =moderate, Score 2 =strong.

\section{Results}

Findings of 12 patients who were diagnosed with CBP (four men and eight women) and operated on in our clinic from January 2000 to January 2021 were analyzed. All demographic, diagnostic, surgical and follow-up results were re-evaluated. Paraffin blocks of ten patients (out of 12) were suitable for PSMA staining and immunohistochemical examination. The age of the patients ranged from 25 to 81 years with a mean of 50 years. The most common initial complaint reported by patients was neck mass. Additionally, four patients had pain, one patient had odynophagia, 
one patient had dysphagia. The time between the initial symptoms and patients' presentation to our clinic ranged from one to 60 months. One patient had bilateral, five had left-sided and six had right-sided CBP. The follow-up period ranged from eight months to ten years. There was no recurrence of the disease in the surgically treated patients (Table 1). Angiographically determined blood supply of the tumors are given in Table 2. Three patients had type I, six patients had type II and three patients had type III tumors according to the Shamblin classification (preoperative and postoperative CT imaging of Shamblin type III CBP are shown in Figures 1a and 1b). In terms of complications, one patient had cerebral emboli that needed embolectomy, and one patient had superior laryngeal nerve paralysis. Carotid artery resection and arterial grafting was needed in only one patient. There was one patient with incomplete resection. Data on complications are shown in Table 2.

\section{PSMA Staining Results}

Intratumoral vascular PSMA expression was strong in eight patients, moderate in one patient. One patient had no PSMA expression (Figures 2 and 3). There was no extratumoral vascular PSMA expression in any of the cases. Tumoral cell PSMA expressions were mild to moderate in six patients and absent in four patients. Comparisons of PSMA expression

Table 1. Demographic features and clinical presentations of patients with CBP

\begin{tabular}{|c|c|c|c|c|c|c|c|c|}
\hline No & Gender & Age & Complaint & DC & Side of the tumor & Family history & Follow-up period & Recurrence \\
\hline 1 & M & 54 & Mass & $6 \mathrm{~m}$ & $\mathrm{~L}$ & No & $3 y$ & No \\
\hline 2 & $\mathrm{~F}$ & 55 & Mass & $48 \mathrm{~m}$ & $\mathrm{R}$ & No & $8 \mathrm{~m}$ & No \\
\hline 4 & M & 52 & Mass & $4 \mathrm{~m}$ & B & No & $2 y$ & No \\
\hline 5 & $\mathrm{~F}$ & 42 & Mass & $6 \mathrm{~m}$ & $\mathrm{R}$ & No & $10 y$ & No \\
\hline 7 & $\mathrm{~F}$ & 62 & Mass & $3 \mathrm{~m}$ & $\mathrm{R}$ & No & $2 y$ & No \\
\hline 8 & $\mathrm{~F}$ & 32 & Mass & $12 \mathrm{~m}$ & $\mathrm{~L}$ & No & $10 y$ & No \\
\hline 9 & $\mathrm{~F}$ & 25 & Mass & $8 \mathrm{~m}$ & $\mathrm{R}$ & No & $10 y$ & No \\
\hline 10 & $\mathrm{~F}^{*}$ & 81 & $\begin{array}{l}\text { Mass, pain, } \\
\text { dysphagia }\end{array}$ & $24 \mathrm{~m}$ & $\mathrm{R}$ & No & $6 y$ & No \\
\hline
\end{tabular}

M: Male, F: Female, m: Months, y: Years, DC: Duration of complaints, R: Right, L: Left, B: Bilateral.

*Patients with tumor embolization before surgery

Table 2. Radiologic and surgical features of patients with CBP

\begin{tabular}{|c|c|c|c|c|c|c|}
\hline No & Size of tumor (mm) & Angiography (blood supply) & Shamblin classification & Complications & Arterial grafting & Incomplete surgery \\
\hline 1 & $28 \times 25 \times 20$ & ECA & I & No & No & No \\
\hline 2 & $31 \times 20 \times 19$ & Ncd & II & No & No & No \\
\hline 3 & $30 \times 25 \times 20$ & ICA & II & Cerebral emboli & No & No \\
\hline 4 & $53 \times 36 \times 35$ & Ncd & II & No & No & No \\
\hline 5 & $38 \times 28 \times 20$ & ECA & II & No & No & No \\
\hline 6 & $57 \times 44 \times 40$ & $\begin{array}{l}\text { Vertebral - pharyngeal artery } \\
\& \text { ICA }\end{array}$ & III & No & No & Yes \\
\hline 7 & $45 \times 36 \times 32$ & Ncd & II & No & No & No \\
\hline 8 & $25 \times 21 \times 20$ & ECA, ICA & III & SLN paralysis & Yes & No \\
\hline 9 & $31 \times 26 \times 11$ & Ncd & I & No & No & No \\
\hline 10 & $55 \times 52 \times 44$ & ICA \& ECA & III & No & No & No \\
\hline 11 & $30 \times 25 \times 17$ & Occipital artery & I & No & No & No \\
\hline 12 & $44 \times 37 \times 34$ & Occipital artery & II & No & No & No \\
\hline
\end{tabular}

ECA: External carotid artery, ICA: Internal carotid artery, SLN: Superior laryngeal nerve, Ncd: Not clearly determined 
with the other immunohistochemical stainings (Ki-67, S100, synaptophysin, chromogranin) and capsular and/or vascular invasion are shown in Table 3.

\section{Discussion}

CBPs or carotid body tumors are also known as chemodectomas because of the carotid body's physiological function as a chemoreceptor (7). Despite the limited number of cases in our study, characteristics such as age, gender and side of tumor were found compatible with the literature $(2$, $8,9)$. CBPs are common in patients that have been exposed to hypoxia for a long time, as well as in those who have chronic obstructive pulmonary disease, congenital cyanotic heart disease, or live at high altitudes (2). The city where
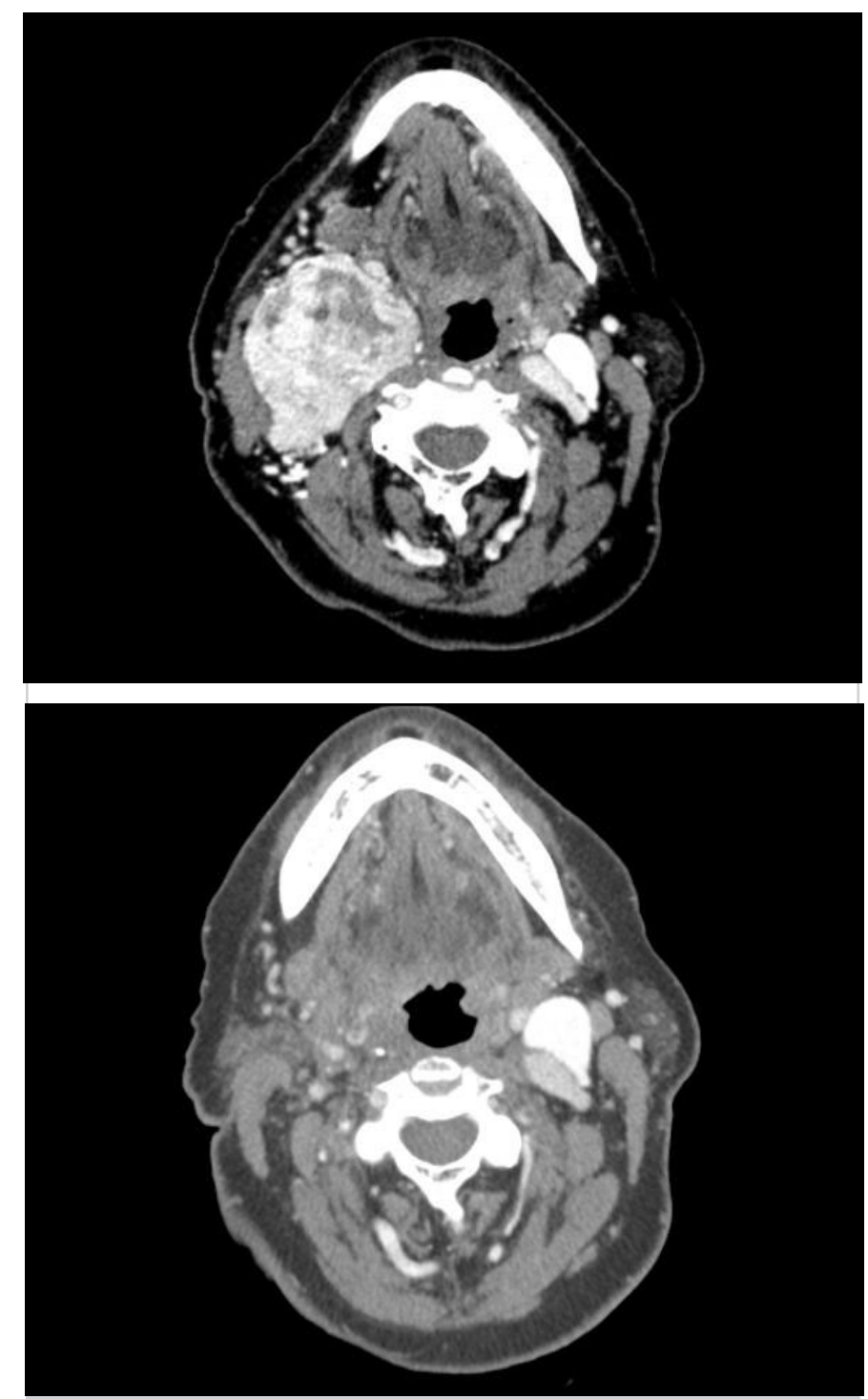

Figure 1. a. Preoperative contrast enhanced CT of the neck demonstrating right-sided Shamblin type III CBP of an 81-yearold female patient; b. Postoperative contrast enhanced CT of the neck

CT: Computed tomography, CBP: Carotid body paraganglioma our patients live is situated at a moderately high altitude of $1,000-1,500$ meters.

The most common complaint of CBP is neck mass. A painless observable pulsatile neck mass, laterally mobile, but vertically immobile, is found only anterior to the sternocleidomastoid muscle, at the plane of the hyoid joint, and under the angle of the mandible on physical inspection (10). The common complaint of all our patients was neck mass. Additional complaints were reported in the clinical course as the tumors grew. Odynophagia and dysphagia were other complaints reported by patients (by one patient each).

The bulk of CBPs are benign, but $6 \%$ are cancerous. Malignancy is defined by the dissemination of cancerous cells to the lymph nodes or distant metastases (7). One out of 12 patients had lymph node metastasis in our study group. Except for one patient, no incomplete surgery or tumor recurrence was observed during the eight-month follow-up period.

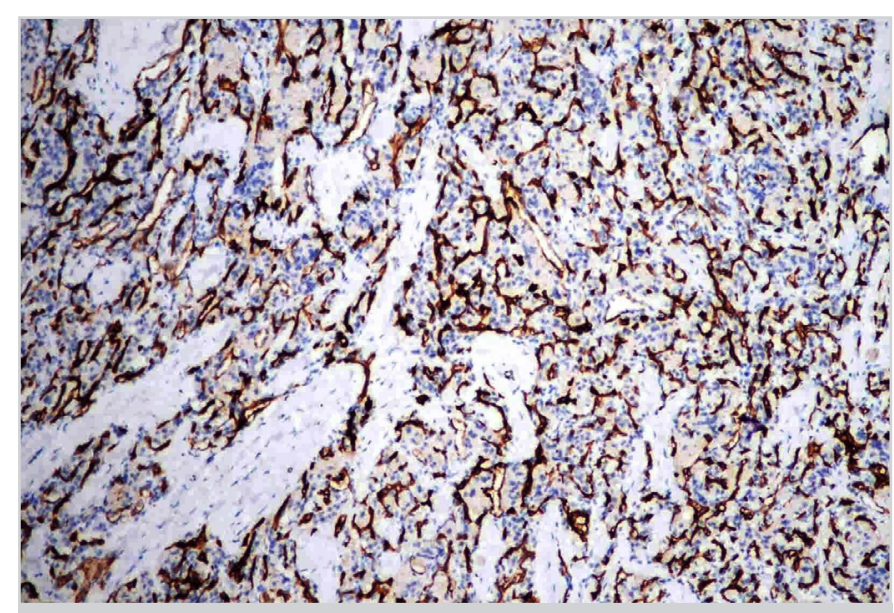

Figure 2. x100 PSMA expression with Score 2 staining in the vessels of the tumoral stroma

PSMA: Prostate-specific membrane antigen

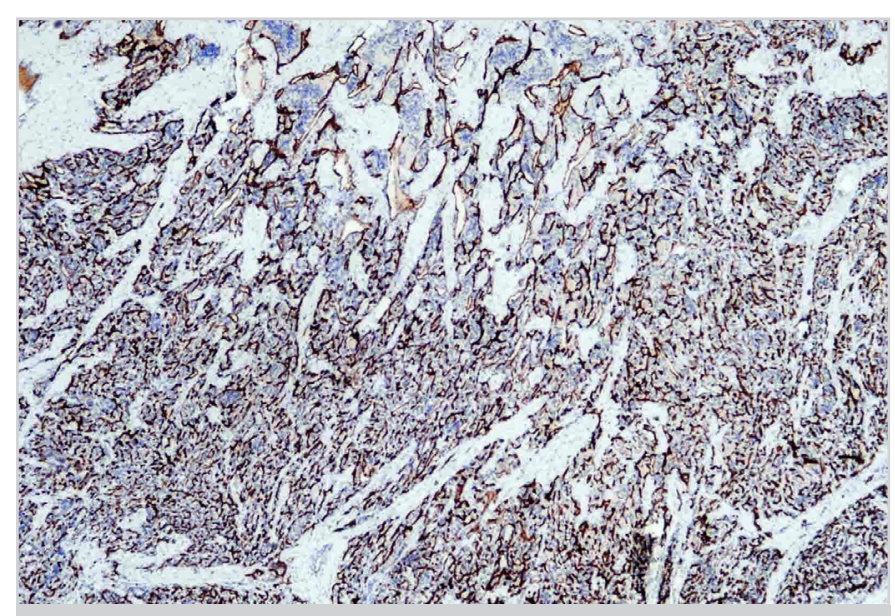

Figure 3. $x 400$ PSMA expression with Score 2 staining in the vessels of the tumoral stroma

PSMA: Prostate-specific membrane antigen 
The gold standard for assessing the vascular function of these tumors is conventional optical subtraction angiography. PGLs are hypervascular masses that splay the internal and external carotid arteries and have major feeding arteries, as well as rapid contrast washout on angiography (11). In our study, all 12 patients underwent angiography. Major feeding vessel(s) were determined immediately before the surgery (Figure 4 shows the angiographic imaging of a right sided $\mathrm{CBP})$. However, only one patient's tumor was successfully embolized.

The Shamblin classification can inform us about the size of the tumor and its relationship to the carotid arteries. Localized tumors with splaying of the carotid bifurcation, but no attachment to the carotid arteries are known as Shamblin Class I CBPs. The carotid vessels are marginally surrounded by Shamblin Class II CBPs, but intimately surrounded by Shamblin Class III CBPs, which are very difficult to dissect completely. It is common for vascular repair to necessitate a temporary disruption in cerebral circulation. In comparison to Class I and Class II, the probability of chronic vascular and neurological deficits is considerably higher in Class III (9). Three of our patients had type I, six had type II and three had type III tumors according to the Shamblin Classification. Incomplete surgery, superior laryngeal nerve paralysis and necessity for arterial grafting were the challenges for these patients. Another aspect worth noting was capsular/vascular invasion seen in the histopathological examination of two out of three patients.

Surgery is the preferred treatment for CBPs, even though it can often be complicated by factors such as carotid artery involvement, tumor vascularity, proximity to cranial nerves, and expansion to the skull base (2). All our patients underwent surgery and only one resulted with incomplete surgery. All patients were followed-up regularly.

Many histopathologic stains, such as chromogranin, synaptophysin, serotonin, and neuron-specific enolase, may be used to differentiate these tumors based on their neuroendocrine nature. These histopathological stains, on the other hand, could be positive for other neural crest tumors,

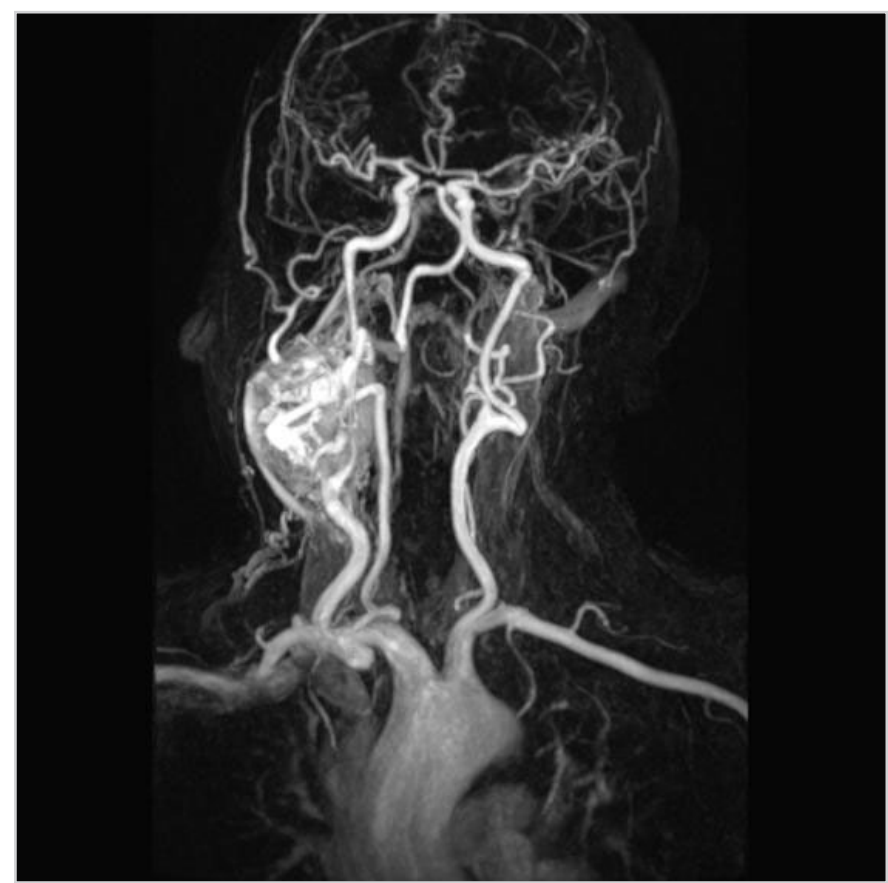

Figure 4. Angiographic appearance of right-sided CBP of 81-yearold female patient

CBP: Carotid body paraganglioma

\begin{tabular}{|c|c|c|c|c|c|c|c|c|c|}
\hline No & $\begin{array}{l}\text { Intratumoral } \\
\text { vascular PSMA } \\
\text { expression }\end{array}$ & $\begin{array}{l}\text { Extratumoral vascular } \\
\text { PSMA expression }\end{array}$ & $\begin{array}{l}\text { Tumoral cell } \\
\text { PSMA staining }\end{array}$ & Ki-67 & S100 & Snp & $\mathrm{Chr}$ & $\mathrm{C} \& \mathrm{~V}$ inv & Met \\
\hline 1 & 2 & 0 & 1 & $5 \%+$ & + & - & - & No & No \\
\hline 2 & 1 & 0 & 1 & $3 \%+$ & + & + & Uir & No & Yes \\
\hline 3 & 2 & 0 & 1 & $3 \%+$ & + & Uir & + & Yes & No \\
\hline 4 & 2 & 0 & 0 & $4 \%+$ & + & - & - & Yes & No \\
\hline 5 & 2 & 0 & 1 & Uir & + & + & + & Uir & No \\
\hline 6 & 2 & 0 & 0 & Uir & Uir & Uir & Uir & Yes & No \\
\hline 7 & 0 & 0 & 0 & $1 \%+$ & + & + & + & No & No \\
\hline 8 & 2 & 0 & 1 & Uir & Uir & Uir & Uir & Yes & No \\
\hline 9 & Upb & Upb & Upb & Uir & + & + & - & Uir & No \\
\hline 10 & 2 & 0 & 0 & $5 \%+$ & + & - & + & No & No \\
\hline 11 & 2 & 0 & 1 & $8 \%+$ & + & + & $?$ & No & No \\
\hline 12 & $\mathrm{Upb}$ & Upb & Upb & Uir & Uir & Uir & Uir & No & No \\
\hline
\end{tabular}

Snp: Synaptophysin, Chr: Chromogranin, C\&V inv: Capsular and/or vascular invasion, Met: Metastasis, Uir: Unexplored in initial reports, Upb: Unsuitable paraffin blocks 
which is a drawback that should be considered before making a differential diagnosis (7).

PSMA is a potential molecule for tumor-specific vascular targeting. PSMA's role in endothelial cell activity is not well understood. According to in vitro and in vivo studies PSMA can play a role in endothelial cell invasion through peptidase activity (4). In case of vascular tumors like $\mathrm{CBPs}$, the investigations of PSMA can provide us new therapeutic and diagnostic ways. Our study has essentially showed the presence of intense PSMA in intratumoral vascular structures. Although tumoral cells do not show strong positivity, their vessels are also major targets for both diagnosis and treatment. Zade et al. (12) determined a neck mass in PSMA-PET/CT compatible with metastasis of prostate cancer. They excised the mass and diagnosed it as glomus jugulare (another PGL of the neck).

According to Foss et al. (13), tumor neovasculature and the neovasculature of certain normal proliferative tissues have higher PSMA expression on the apical and luminal surface of new blood endothelial cells (endometrium, heart valve injuries, pleural lesions, and keloid scars). All these findings show the importance of PSMA in the neovascularization and blood supply of the solid tumors.

In vivo PSMA expression in tumors can serve as a replacement molecule for neovascularization, according to Tripathy et al. (5), and this concept can be used for selective therapy in vascular benign tumors such as head and neck PGLs if resection is difficult or impossible. Our study revealed that strong expression of PSMA occurred in the intratumoral vascular structures of CBPs. PSMA targeted prospective studies (either diagnostic or therapeutic) may be planned according to intratumoral vascular expression of PSMA.

The limitations of our study were mainly its preliminary nature and limited numbers of cases: While the number of female patients was twice that of male patients, the small number of patients did not allow us to reach a conclusion about the effect of gender on PSMA expression. The same limitation exists for age groups. More comprehensive studies involving larger numbers of patients are required to overcome these limitations.

\section{Conclusion}

We detected PSMA expression in CBPs. The most important part of this expression is intratumoral vascular structures. We did not find PSMA expression in the extratumoral vascular structures. We recommend conducting prospective studies targeting intratumoral vascular PSMA expression. PSMA targeted modalities may be the future of diagnostic and therapeutic methods. This needs to be confirmed by prospective studies.

Ethics Committee Approval: The Clinical Research Ethics Committee of Süleyman Demirel University approved our study protocol. (approval date: 11/09/2020; approval number: 254).

Informed Consent: The study was designed as a retrospective study; therefore, informed consent was not obtained from the patients.

Peer-review: Externally peer-reviewed.

Conflict of Interest Statement: The authors declare no conflict of interest.

Financial Disclosure: This study was funded by The Coordinatorship of Scientific Research Projects Department (BAP), Süleyman Demirel University by the number of TSG-2020-8134.

\section{Authorship Contributions}

Surgical and Medical Practices: H.Y., E.O., Concept: H.Y., Y.Ç.K., M.E.S., Design: H.Y., M.E.S., E.O., Data Collection and/or Processing:Y.Ç.K., İ.M.Ç., Analysis and/ or Interpretation: H.Y., Y.Ç.K., İ.M.Ç., M.E.S., Literature Search: H.Y., İ.M.Ç., E.O., Writing: H.Y., Y.Ç.K., M.E.S., E.O.

\section{Main Points}

- The most common paragangliomas in the head-neck region are $\mathrm{CBPs}$, and they are primarily treated with surgery.

- For CBPs, new treatment strategies can be developed in cases which surgery cannot be performed.

- The presence of PSMA has been detected in the neovasculature of some non-prostatic solid tumors.

- This study has shown the presence of PSMA in CBPs, and PSMA is expected to shed light on new treatment strategies.

\section{References}

1. Scholzen T, Gerdes J. The Ki-67 protein: from the known and the unknown. J Cell Physiol 2000; 182: 311-22. [Crossref]

2. Melachuri S, Valappil B, Snyderman C. Variations in surgical outcomes of carotid body tumors by surgical specialty. Laryngoscope 2021; 131: 190-5. [Crossref]

3. Williams MD. Paragangliomas of the head and neck: an overview from diagnosis to genetics. Head Neck Pathol 2017; 11: 278-87. [Crossref]

4. Haffner MC, Laimer J, Chaux A, Schäfer G, Obrist P, Brunner A, et al. High expression of prostate-specific membrane antigen in the tumor-associated neo-vasculature is associated with worse 
prognosis in squamous cell carcinoma of the oral cavity. Mod Pathol 2012; 25: 1079-85. [Crossref]

5. Tripathy S, Tripathi M, Dattagupta S, Parida GK, Shamim SA. In vivo PSMA expression in head and neck paragangliomas on $68 \mathrm{Ga}$ PSMA 11 PET/CT. Clin Nucl Med 2019; 44: 398-400. [Crossref]

6. Choudhury PS, Gupta M. Theranostics in India: a particularly exquisite concept or an experimental tool. Nucl Med Mol Imaging 2019; 53: 92-5. [Crossref]

7. Fathalla AE, Elalfy MA. Clinical outcome of carotid body paraganglioma management: a review of 10-year experience. J Oncol 2020: 6081273. [Crossref]

8. Rashaideh MA, Neshwati FM, As'ad M, Ajarmeh E, Alsharayeh $\mathrm{E}$, Yasin N, et al. Diagnosis and treatment of carotid body tumors: a retrospective analysis of a single-center experience over 12 years. Indian J Vasc Endovasc Surg 2020; 7: 34-8. [Crossref]
9. Offergeld C, Brase C, Yaremchuk S, Mader I, Rischke HC, Gläsker S, et al. Head and neck paragangliomas: clinical and molecular genetic classification. Clinics (Sao Paulo) 2012; 67: 1928. [Crossref]

10. Jianu DC, Jianu SN, Motoc AG, Dan TF, Poenaru M, Tăban S, et al. An evaluation on multidisciplinary management of carotid body paragangliomas: a report of seven cases. Rom J Morphol Embryol 2016; 57: 853-9. [Crossref]

11. Baz RA, Scheau C, Sârbu N, Costea DO, Dijmărescu A, Bordei P. Carotid paragangliomas: case report and imaging review. Folia Morphol (Warsz). 2021; 80: 699-706. [Crossref]

12. Zade A, Trivedi C, Ingule A, Jha S, Agrwal A.Incidentally detected 18F-PSMA uptake in glomus jugulare. Clin Nucl Med 2020; 45: 360-2. [Crossref]

13. Foss CA, Mease RC, Cho SY, Kim HJ, Pomper MG. GCPII imaging and cancer. Curr Med Chem 2012; 19:1346-59. [Crossref] 